\title{
Hubbard model versus $t-J$ model: The one-particle spectrum
}

\author{
Henk Eskes \\ Institute Lorentz, Leiden University, P.O. Box 9506, 2300 RA Leiden, The Netherlands \\ Robert Eder \\ Laboratory of Applied and Solid-State Physics, Materials Science Centre, \\ University of Groningen, Nijenborgh 4, 9747 AG Groningen, The Netherlands
}

(February 6, 2008)

\begin{abstract}
The origin of the apparent discrepancies between the one-particle spectra of the Hubbard and $t-J$ models is revealed: Wavefunction corrections, in addition to the three-site terms, should supplement the bare $t-J$ model. In this way a quantitative agreement between the two models is obtained, even for the intermediate- $U$ values appropriate for the high- $T_{c}$ cuprate superconductors. Numerical results for clusters of up to 20 sites are presented. The momentum dependence of the observed intensities in the photoemission spectra of $\mathrm{Sr}_{2} \mathrm{CuO}_{2} \mathrm{Cl}_{2}$ are well described by this complete strongcoupling approach.
\end{abstract}

71.27.+a,79.60.-i,71.10.+x,74.72.-h

The past ten years the interest in Hamiltonians modeling correlated electrons has greatly increased. This is largely due to the mysterious normal-state properties of the high- $T_{c}$ cuprate superconductors and the realization that local correlations on the $\mathrm{Cu}$ sites are large in these compounds. The two most intensively studied Hamiltonians are the Hubbard and $t-J$ models. Although at first sight such models may seem oversimplified, it has been argued that the two dimensional square-lattice Hubbard model captures the essential dynamics of the cuprates in a window of a few $\mathrm{eV}$ around the chemical potential [1]]. The materials belong to the intermediate- $U$ regime, $U / t \approx 10, t \approx 0.3-0.4 \mathrm{eV}[3,, 4]$. Further support for a single-band Hubbard approach comes from measured one-particle and optical spectra of the cuprates. These show a dramatic reshuffling of intensity as a function of doping on the scale of a few eV [5, 6]. This characteristic behavior is a fingerprint of strong correlations and is described well by the Hubbard model [5, [7.8].

Although the $t$ - $J$ model is frequently referred to as being the large- $U$ limit of the single-band Hubbard model, the (one-particle) spectra of the two models differ significantly in the intermediate- $U$ regime of interest. (For a recent review on numerical results, see Ref. [7]) Probably because of this, the two models are often treated as being "distinct". One obvious difference concerns the momentum occupation number $n_{\mathbf{k}}$ at half filling, equal to the one-electron removal sum rule. For the $t-J$ model this quantity is trivially equal to $1 / 2$, while for the intermediate- $U$ Hubbard model it is a strongly varying function of $\mathbf{k}$. Even though in the infinite- $U$ limit $n_{\mathbf{k}}$ tends to $1 / 2$, as in the $t$ - $J$ model, the convergence to this value by increasing $U$ is very slow.

In this Letter we show that these discrepancies can be attributed to the neglect of certain terms in the large$U$ perturbation theory. Specifically, the weight distributions in the spectral functions show a high sensitivity to the first-order renormalization of the wave functions. The complete strong-coupling approach is found to quantitatively reproduce the Hubbard spectra for $U \geq W$, where $W$ is the $U=0$ band width. For these $U$ values the perturbation series in $t / U$ agrees well with the full Hubbard Hamiltonian.

The derivation of the strong-coupling Hamiltonian by means of a canonical transformation, written as an expansion in $t / U$, is well known [8,9]. Starting point is the Hubbard model,

$$
H=V+T=U \sum_{i} n_{i, \uparrow} n_{i, \downarrow}-t \sum_{i, \delta, \sigma} a_{i, \sigma}^{\dagger} a_{i+\delta, \sigma} .
$$

Here $n_{i, \uparrow}$ is the occupation number of the original Hubbard fermions $a_{i, \sigma}$, and $\delta$ is a vector connecting nearest neighbors. After the transformation the states in the lowest Hubbard sector (no doubly occupied sites) are described to order $t^{2} / U$ by the strong-coupling Hamiltonian,

$$
\begin{aligned}
& H_{s c}=-t \sum_{i, \delta, \sigma} c_{i, \sigma}^{\dagger} c_{i+\delta, \sigma}-\frac{J}{2} \sum_{i, \delta}\left(\tilde{\mathbf{S}}_{i} \cdot \tilde{\mathbf{S}}_{i+\delta}-\frac{1}{4} \tilde{n}_{i} \tilde{n}_{i+\delta}\right) \\
& -\frac{J}{4} \sum_{i, \delta \neq \delta^{\prime}, \sigma}\left(c_{i+\delta, \sigma}^{\dagger} \tilde{n}_{i, \bar{\sigma}} c_{i+\delta^{\prime}, \sigma}-c_{i+\delta, \bar{\sigma}}^{\dagger} c_{i, \sigma}^{\dagger} c_{i, \bar{\sigma}} c_{i+\delta^{\prime}, \sigma}\right),
\end{aligned}
$$

where $J=4 t^{2} / U$. The motion of the above transformed $c_{i, \sigma}$ fermions will leave the number of doubly occupied sites unchanged, and $\tilde{\mathbf{S}}_{i}$ and $\tilde{n}_{i, \sigma}$ are the spin and occupation number of these new fermions. Simply neglecting the 'three-site term', i.e. the second line of (2), one obtains the $t-J$ model.

Why the full strong-coupling model has received far less attention than the $t-J$ model is unclear. It might be expected that the three-site term is very important in the one-hole limit (and low doping region) since, unlike the 
nearest-neighbor hopping, a direct next-nearest-neighbor hopping process does not frustrate the spin system. However, it was shown by a numerical study [10], spin-polaron approaches 11.12] and a linear-spin-wave self-consistent Born (LSW-SCB) calculation [13] that the main effect of this term on the quasiparticle dispersion is an increase of the bandwidth of the dominant 'quasiparticle peak'.

By virtue of its construction via a canonical transformation, the Hamiltonian (2) reproduces the eigenvalue spectrum of the Hubbard model for large $U$. To obtain further information, such as matrix elements or expectation values, operators acting in the Hilbert space of the Hubbard model have to be subjected to the same canonical transformation. For the electron annihilation operator this procedure leads to the expression [8,9],

$$
\begin{aligned}
& P a_{i, \sigma} P=P c_{i, \sigma} P \\
& \quad+\frac{t}{U} \sum_{\delta} P\left(\tilde{n}_{i, \bar{\sigma}} c_{i+\delta, \sigma}-c_{i, \bar{\sigma}}^{\dagger} c_{i, \sigma} c_{i+\delta, \bar{\sigma}}\right) P .
\end{aligned}
$$

$P$ is defined as the projector on states with no double occupancy of the transformed fermions (states with $\langle\tilde{V}\rangle=0$ ). The first-order correction terms (second line) describe the dressing of the annihilation operator with 'virtual' charge fluctuations involving doubly-occupied sites. While their prefactor $t / U$ seems to suggest that they represent only a minor correction, their impact on the calculated spectra is dramatic - one reason being that the summation over neighbors introduces the coordination number $z$ as an additional 'hidden' prefactor. When comparing strong-coupling calculations directly with experiments it is therefore crucial to take these corrections into account. For the optical conductivity such corrections were shown to lead to qualitatively different results [14]. Another consequence is a rapid reshuffling of weight between the upper (UHB) and lower-Hubbard band (LHB) as a function of hole doping, observed in both one-particle and optical experiments. The first-order corrections to transformed operators such as Eq. (3) lead to modified expressions for sum rules that consistently describe the rapid intensity changes observed in experiments [5, 6, 8,9 .

For the numerical calculations of the one-particle spectra we implemented the strong-coupling model Eq. (2). Electron removal is described by the operator Eq. (3). This operator does not change double-occupancy and only the LHB part of the Hubbard spectrum is produced. (There is, in fact, a similar independent procedure to calculate the UHB response as well.) The $t / U$ term in Eq. (3) amounts to the removal of an electron from a neighbor of $i$. This leads to an extra phase factor $\exp (i \mathbf{k} \cdot \delta)$. Because of this the total weight is enhanced at $\Gamma$ and reduced at $(\pi, \pi)$ and Eq. (3) leads to the correct behavior of $n(\mathbf{k})$ (see Fig. 2 of Ref. [8]). Note that the implementation of the first-order correction is straightforward and does not increase memory or CPU time in

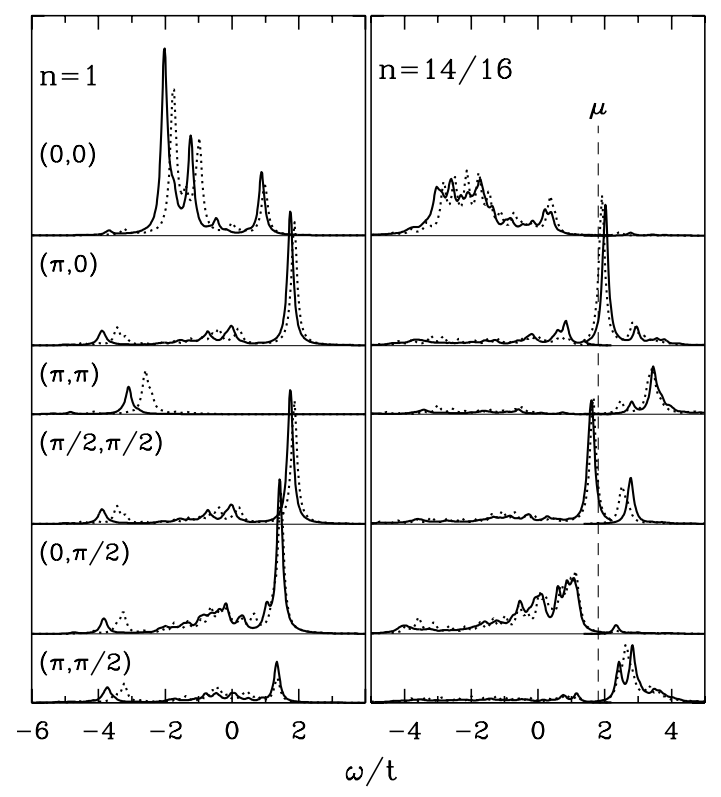

FIG. 1. The one-particle spectrum of the two-dimensional $4 \times 4$ strong-coupling model (solid line) compared with the Hubbard model (dotted line), at half filling (left) and for two holes (right). $U / t=10$. Only the LHB part of the Hubbard spectrum is shown. Dashed line is the chemical potential in the two-hole case. Hubbard data reproduced from Ref. 15.

a Lanczos-like diagonalization calculation. In a similar way such operator corrections can be taken into account in analytic approaches, for instance in the LSW-SCB calculation of the one-hole spectrum.

In Fig. 1 we compare the two-dimensional 16-site 4x4 strong-coupling model with the LHB electron removal and addition spectra of the Hubbard model, reproduced from Ref. 15]. Note that $U=10$ is comparable to the bandwidth $W=8$. No shift or scaling has been applied to the spectra, showing that the value of the gap between the UHB (not shown, starting at energy $\approx 8 t$ ) and LHB is accurately described. (While there is no UHB in the strong coupling model, the magnitude of the energy gap still can be inferred from the relation $\Delta=U-2 \cdot E_{\text {min }}$, with $E_{\text {min }} \approx 2 t$ the minimal ionization energy of the model.) The resulting gap of $\approx 6 t$ is consistent with the measured insulating gap of $\approx 2 \mathrm{eV}$.

As can be seen, excitation energies and weights of the dominant features in the spectra are reproduced correctly by the strong-coupling approach. The agreement is best for the physically most relevant low excitation energies, but the strong coupling approach also gives a complete description of the higher excitations, with energies not much smaller than $U$. The total width is slightly overestimated by the second-order $t^{2} / U$ terms in the Hamiltonian, due especially to those peaks which obtain a large energy correction by the three-site terms, for instance at $(\pi, \pi)$. There is no significant deterioration of the agreement upon doping, demonstrating that the discrepancies 
between the doping dependences of low energy sum-rules for $t-J$ and Hubbard originate from matrix element effects, rather than an incompleteness of the Hilbert space of the strong coupling model. We explicitly checked that second-order corrections to the Fermi operators lead to only minor changes of the peak intensities. For $U$ values below 10 the agreement between Hubbard and its strongcoupling limit gradually deteriorates, which we interpret as a breaking down of the perturbation approach.

Stimulated by the good agreement in the $4 \mathrm{x} 4$ system we now present large-U Hubbard spectra for clusters up to 20 sites. Because the momenta of the 16 and 18 site clusters nicely complement each other and are all on the high-symmetry axis, we show a mix of these two clusters in Figs. 2 and 3. The curves on the left show a scan from $(0,0)$ to $(\pi, \pi)$. The plots on the right go from $(0,0)$ to $(\pi, 0)$ and from $(\pi, 0)$ to $(\pi, \pi)$. We shifted all $4 \times 4$ spectra by the same small energy such that the lowest peak at $\mathbf{k}=(0,0)$ is at the same energy for both clusters. For the two-hole case we shifted the $t-J$ data by an additional $0.3 t$ to have the chemical potentials at roughly the same position. The ground-state momentum is $(0,0)$ in all cases (there is an extra degeneracy for 16-sites, 2 holes). In both figures the results are compared with the "bare" $t-J$ model.

As mentioned before there are substantial differences between $t$ - $J$ and strong coupling. At half-filling the low energy 'quasiparticle peaks' are at higher energies (implying a larger Hubbard gap) for the

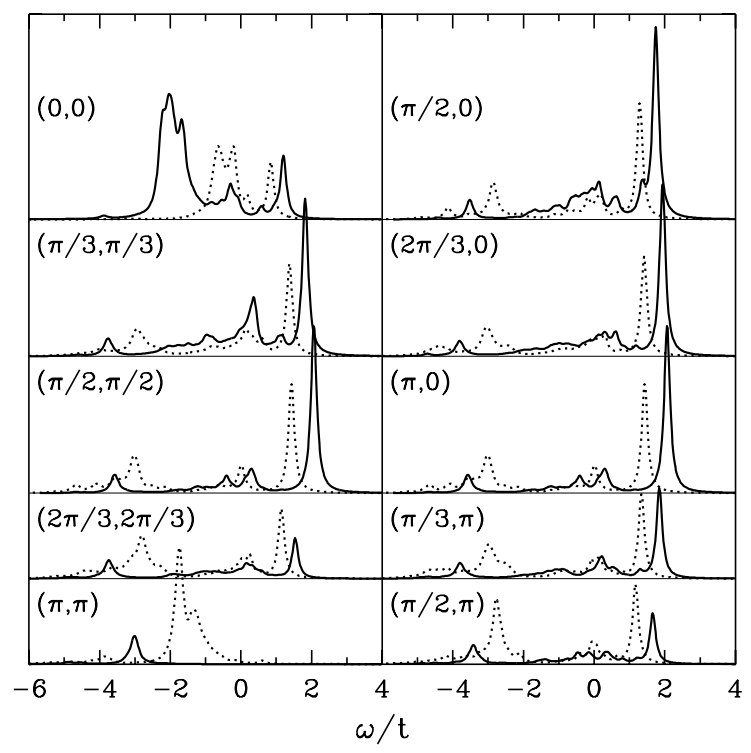

FIG. 2. The momentum dependence of the electron-removal spectrum of the strong-coupling Hubbard model (solid line) compared with the $t$ - $J$ model (dotted line). The plot is a mix of data from the 16 and 18 site clusters. $U / t=10 . n=1$. The spectra for $\mathbf{k}=(\pi / 2, \pi / 2),(\pi / 2,0)$, $(\pi, 0)$ and $(\pi / 2, \pi)$ are calculated using the $2 \mathrm{D} 16$-site cluster; the others, using the 18 -site cluster.

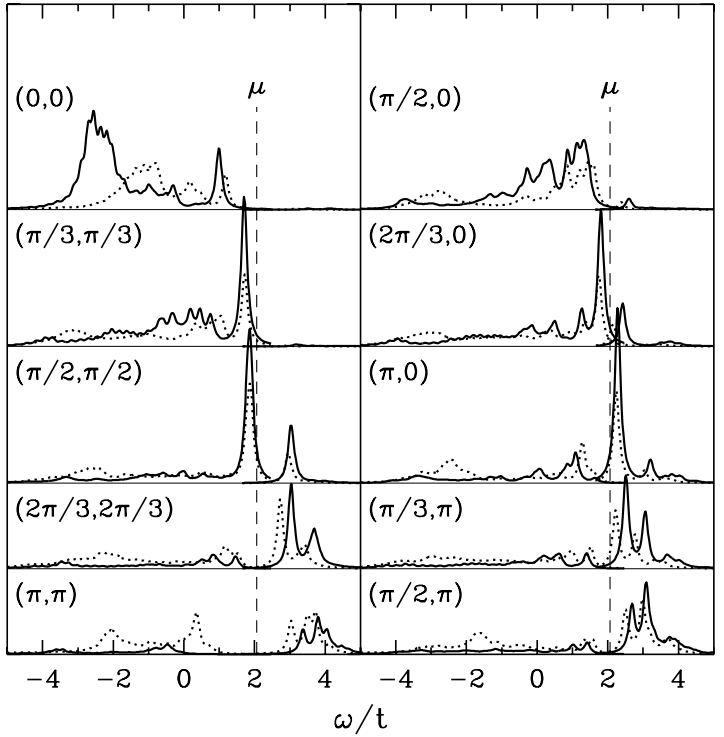

FIG. 3. Combined electron addition and removal spectra for the two-hole ground state. See caption of Fig. 2.

$t-J$ model and their dispersion has a smaller width. Moreover, the momentum dependence of the weights is qualitatively different. The $t-J$ spectrum at $(0,0)$, and indeed through much of the Brillouin zone, is more compact than for large- $U$ Hubbard. The strongest difference is at $(\pi, \pi)$. Here strong coupling shows a little peak at high energy, while the $t-J$ model has pronounced structures at a more intermediate energy. This reflects the small value of $n_{\mathbf{k}}$ for $\mathbf{k}=(\pi, \pi)$ in the Hubbard model, which is reproduced by the first-order corrections to the Fermi operator Eq. (3).

The spectra at half filling can be compared with recent angle-resolved photoemission data on insulating $\mathrm{Sr}_{2} \mathrm{CuO}_{2} \mathrm{Cl}_{2}$ [16. A significant feature of these measurements is the strong momentum dependence of the weights of the spectra. The region in momentum space with the most intense peaks is not centered around $(\pi / 2, \pi / 2)$, but displaced towards $(0,0)$. Beyond $(\pi / 2, \pi / 2)$ the intensity drops very sharply and seems to vanish around $(\pi, \pi)$ [17. As shown in Fig. 4 these features are well reproduced by the strong-coupling Hubbard model. In contrast, the intensities in $t-J$ are much more symmetric around $(\pi / 2, \pi / 2)$ and change only little in that part of momentum space. On average the peaks in strongcoupling Hubbard are more intense than those in the $t-J$ model, at the expense of the incoherent part of the spectrum. Note that also in the $t-J$ model there are differences in weight between $\mathbf{k}$ and $\mathbf{k}+(\pi, \pi)$. This is due to the coupling of the created hole to quantum-spin fluctuations in the ground state of the Heisenberg antiferromagnet [12]. Experimentally the strong changes at the antiferromagnetic Brillouin-zone boundary look like 

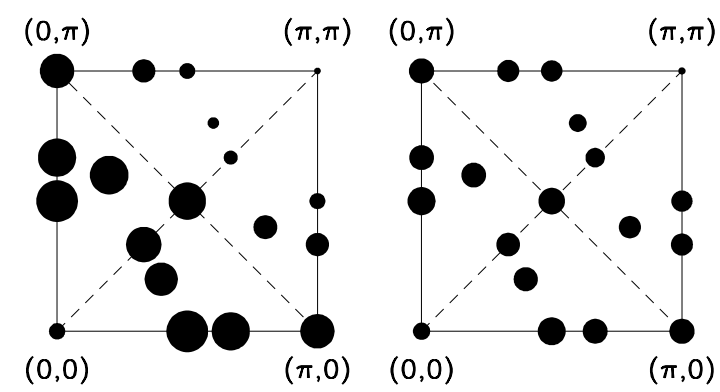

FIG. 4. Weights (proportional to the radius of the circles) of the lowest energy peak for the strong-coupling Hubbard model (left) and $t-J$ model (right). Data collected from the 16,18 and 20 site clusters. $U / t=10, n=1$.

a kind of 'pseudo Fermi surface': The spectral weight observed drops significantly, but an actual Fermi-level crossing does not take place. We note that the large difference observed between $(\pi, 0)$ and $(\pi / 2, \pi / 2)$ in the photoemission measurements actually is in disagreement with the near degeneracy of these momenta in both the standard Hubbard and $t-J$ model. As discussed by various authors this may be explained by adding extra nextneighbor hopping terms to the Hamiltonian [13,18].

The increased quasiparticle weight and the strong drop of intensity when passing the half-filled Fermi surface are consistent with a continuous cross-over to a small- $U$ spin-density-wave (SDW) like shadow-band scenario 19. Surely Fig. 1 shows that such a strong drop in weight is not necessarily a weak-coupling feature.

The interpretation of the doping dependence of the spectral function is a subtle issue 20 23], and will not be discussed here. For roughly 10\% holes (Fig. 3) there are intense features appearing in the electron addition spectrum for all momenta outside the magnetic zone. These features are more intense than for $t-J$ and because of the sum rule the extra weight must come from the upper Hubbard band. This weight-transfer effect has been clearly observed in O-1s X-ray absorption [5].

In conclusion, we have shown that the strong-coupling approach is a good alternative to Hubbard calculations even in the intermediate $U$ regime relevant for the high$T_{c}$ superconductors. Only the complete strong-coupling model gives a faithful representation of the properties of the Hubbard model. The corrections to $t-J$ lead to a smooth crossover to small $U$. For comparisons with experiments such as angle-resolved photoemission, O-1s X-ray absorption (and optical conductivity) it is important to use the renormalized wavefunctions (rotated operators), as these can lead to even qualitatively different results.
We acknowledge stimulating discussions with George A. Sawatzky, Jan Zaanen and Andrzej M. Oleś. HE is supported by the Stichting voor Fundamenteel Onderzoek der Materie (FOM), which is financially supported by the Nederlandse Organisatie voor Wetenschappelijk Onderzoek (NWO). RE is supported by a postdoctoral fellowship granted by the Commission of the European Communities.

[1] P.W. Anderson, Science 235, 1196 (1987).

[2] F.C. Zhang and T.M. Rice, Phys. Rev. B 37, 3757 (1988).

[3] M.S. Hybertsen et al., Phys. Rev. B 41, 11068 (1990).

[4] L.F. Feiner, J.H. Jefferson, and R. Raimondi, Phys. Rev. B 53, 8751 (1996), and references therein.

[5] C.T. Chen et al., Phys. Rev. Lett. 66, 104 (1991).

[6] S. Uchida et al., Phys. Rev. B 43, 7942 (1991);

[7] E. Dagotto, Rev. Mod. Phys. 66, 763 (1994), and references therein.

[8] H. Eskes and A. M. Oleś, Phys. Rev. Lett. 73, 1279 (1994); H. Eskes et al., Phys. Rev. B 50, 17980 (1994).

[9] A.B. Harris and R.V. Lange, Phys. Rev. 157, 295 (1967).

[10] K.J. von Szczepanski, et al., Phys. Rev. B 41, 2017 (1990).

[11] S.A. Trugman, Phys. Rev. B 37, 1597 (1988); ibid. Phys. Rev. B 41, 892 (1990).

[12] R. Eder and K.W. Becker, Z. Phys. B 78, 219 (1990); ibid. Phys. Rev. B 44, 6982 (1991).

[13] J. Bała, A.M. Oleś and J. Zaanen, Phys. Rev. B 52, 4597 (1995).

[14] W. Stephan and P. Horsch, Int. J. Mod. Phys. B6, 141 (1992). P. Horsch and W. Stephan, Phys. Rev. B 48, 10595 (1993).

[15] P.W. Leung et al., Phys. Rev.B 46, 11779 (1992).

[16] B.O. Wells et al., Phys. Rev. Lett. 74, 964 (1995).

[17] It is important to realize that other effects can influence the momentum dependence of intensities as well, such as the changing angle of the escaping electron and the actual underlying $\mathrm{Cu}-3 \mathrm{~d}, \mathrm{O}-2 \mathrm{p}$ orbital structure.

[18] See, for instance, A. Nazarenko et al., Phys. Rev. B 51, 8676 (1995).

[19] A.R. Kampf and J.R. Schrieffer, Phys. Rev. B 42, 7967 (1990).

[20] W. Stephan and P. Horsch, Phys. Rev. Lett. 66, 2258 (1991).

[21] R. Preuss, W. Hanke, and W. von der Linden, Phys. Rev. Lett. 75, 1344 (1995).

[22] R. Eder, Y. Ohta, and T. Shimozato, Phys. Rev. B 50, 3350 (1994); R. Eder and Y. Ohta, to appear in Phys. Rev. B.

[23] X.G. Wen and P.A. Lee, Phys. Rev. Lett. 76503 (1996). 